\title{
Synthetic Post-AGB Evolution
}

\author{
Adam Frankowski \\ N. Copernicus Astronomical Center, ul. Rabiańska 8, 87-100 Toruń, \\ Poland
}

\begin{abstract}
A synthetic method for tracing the evolution of the postAGB stars is presented. The main advantage of this procedure, based on the published numerical evolutionary sequences, is that it is simple, fast, and allows to treat post-AGB objects of arbitrary mass.
\end{abstract}

\section{Introduction}

The post-AGB stage of stellar evolution is recently a subject of intense studies both observational and theoretical. Unfortunately, there exist few detailed, timedependent, numerical models of a star evolving through this transient phase between AGB and PN stages. These models are calculated only for a very limited number of progenitor masses. The main reason for this situation is the complexity of the problem and high computational cost of such modelling. But in some applications, eg. in the population synthesis, it is essential to trace post-AGB evolution of many objects of different initial mass. This means that a fast, synthetic method of calculating the post-AGB evolution is needed. Here I present such a method. The proposed procedure allows to treat post-AGB objects of arbitrary mass without excessive numerical costs.

Paczyński (1971) has shown that the evolution of a star from a red giant to PN stage can be conveniently presented on a diagram displaying the hydrogenrich envelope mass vs. the effective temperature. The $M_{\text {env }}-T_{\text {eff }}$ diagram has later been used to describe the post-AGB behavior by Schönberner $(1981 ; 1983)$ and Blöcker (1995). On such a diagram the star spends its AGB phase in the low temperature, high envelope mass region, with the effective temperature generally decreasing as the envelope mass decreases. The departure from the AGB begins when this trend is reversed. Then, the post-AGB phase shows up as a sharply downward and then quasi-horizontal line, going from low to high effective temperatures with decreasing envelope mass. At $\mathrm{T}_{\text {eff }} \sim 3 \times 10^{4} \mathrm{~K}$ the stellar radiation starts to ionize the ejected matter and a $\mathrm{PN}$ is formed.

The post-AGB phase is short $\left(\leq 10^{4} \mathrm{yr}\right)$ and proceeds at nearly constant CO core mass. In this short time the mass of the hydrogen-rich envelope is reduced by orders of magnitude and $\mathrm{T}_{\text {eff }}$ increases dramatically. Hence the $\mathrm{M}_{\text {env }}$ vs. $\mathrm{T}_{\text {eff }}$ relation in the post-AGB phase can be treated as characterized by a specific $\mathrm{CO}$ core mass, $M_{\text {core }}$. This fact has been used to construct a $M_{\text {core-parametrized }}$ synthetic $\mathrm{M}_{\text {env }}-\mathrm{T}_{\text {eff }}$ relation that allows to trace the evolution of a post-AGB object of arbitrary core mass. When accompanied with some wind formula, this relation gives also the time evolution of stellar parameters. 


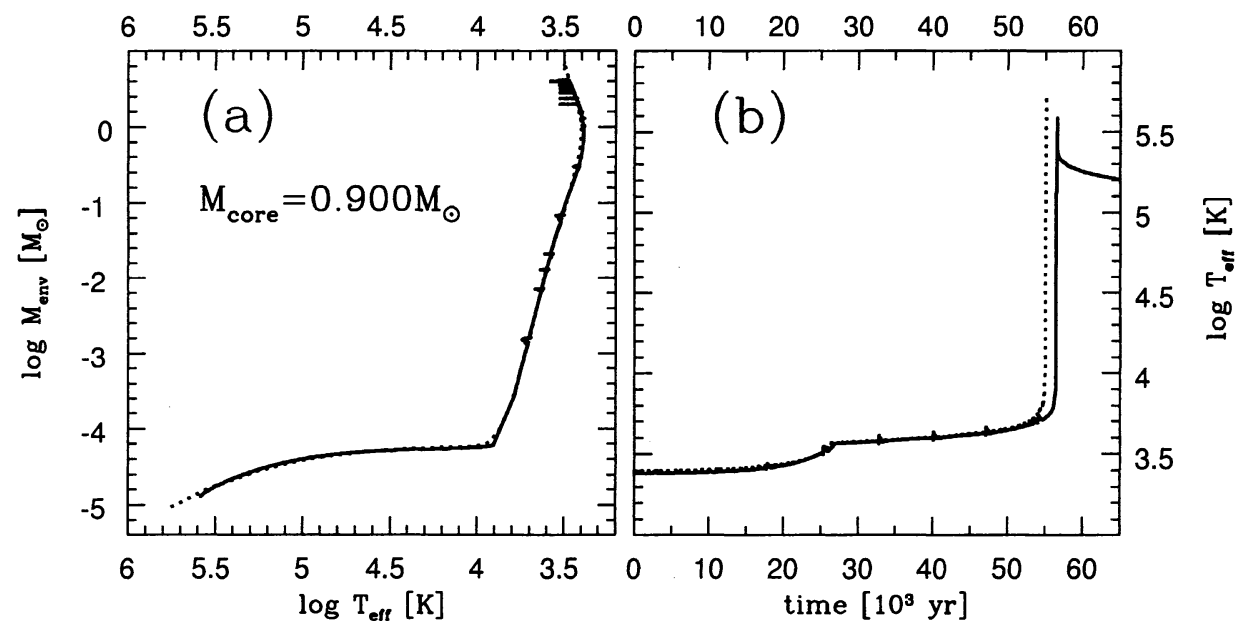

Figure 1. A comparison of numerical (solid line) and synthetic (dotted line) results for a post-AGB star of $\mathrm{M}_{\text {core }}=0.9 \mathrm{M}_{\odot}$ : (a) $\mathrm{M}_{\text {env }}-\mathrm{T}_{\text {eff }}$ diagram, (b) temporal evolution of the effective temperature.

\section{Sample results}

The $\mathrm{M}_{\text {core-parametrized }} \mathrm{M}_{\text {env }}-\mathrm{T}_{\text {eff }}$ relation presented here has been obtained by the analysis of $\mathrm{M}_{\text {env }}$ vs. $\mathrm{T}_{\text {eff }}$ diagrams derived from evolutionary tracks of Vassiliadis \& Wood $(1993 ; 1994)$. In the proposed parametrization the shape of the synthetic relation and its position on the $\mathrm{M}_{\text {env }}-\mathrm{T}_{\text {eff }}$ plane is for a given core mass determined by several coefficients having simple dependance on $M_{\text {core }}$.

Figure 1 presents as an example the results of the synthetic modelling for an object of core mass of $0.9 \mathrm{M}_{\odot}$, compared to the original numerical track of Vassiliadis \& Wood of the same core mass. In each panel solid lines correspond to the numerical results, dotted - to the synthetic ones. Fig. 1a shows numerical and synthetic $\mathrm{M}_{\mathrm{env}}-\mathrm{T}_{\text {eff }}$ diagrams. Fig. $1 \mathrm{~b}$ compares the time evolution of the effective temperature in the original numerical track and as reproduced by the proposed synthetic method for the core mass. The mass loss prescription in the synthetic calculations is the same as used by Vassiliadis \& Wood.

Acknowledgments. This work has been supported from the grant No. 5.P03D.005.21 of the Polish State Committee for Scientific Research

\section{References}

Blöcker, T. 1995, A\&A, 299, 755

Paczyński, B. 1971, AcA, 21, 41

Schönberner, D. 1981, A\&A, 103, 119

Schönberner, D. 1983, ApJ, 272, 708

Vassiliadis, E. \& Wood, P.R. 1993, ApJ, 413, 641

Vassiliadis, E. \& Wood, P.R. 1994, ApJS, 92, 125 